\title{
Macroglossia como Primeira Manifestação Clínica da Amiloidose Primária
}

\section{Macroglossia as Initial Clinical Manifestation of Primary Amyloidosis}

\author{
Carla de Oliveira Alambert ${ }^{(1)}$, Maíra de Oliveira Sarpi ${ }^{(2)}$, Rogério Aparecido Dedivitis ${ }^{(3)}$, Paulo Augusto \\ Alambert $^{(4)}$, Ângelo Sementilli ${ }^{(5)}$, Luciana Pierotti Arantes ${ }^{(6)}$
}

\section{RESUMO}

A amiloidose é uma doença rara causada pelo depósito patológico de substância amilóide no meio extracelular. Relatamos o caso de um paciente de 50 anos de idade, masculino, com história de edema de lábios e língua associado à equimose periorbitária bilateral há 6 meses. Ao exame físico, observamos importante macroglossia. Foram realizados exames complementares, e o diagnóstico foi confirmado pela análise histopatológica da biópsia de língua. O paciente recebeu tratamento com prednisona e melfalan com resposta insatisfatória. Evoluiu com insuficiência cardíaca congestiva e infecções urinárias e respiratórias de repetição. Após 7 meses do diagnóstico, o paciente foi internado com infecção pulmonar complicada com sepse e evoluiu a óbito.

Palavras-chave: amiloidose, macroglossia, equimose periorbital.

\section{INTRODUÇÃO}

A amiloidose é uma doença rara de diagnóstico difícil. Isso ocorre porque as primeiras manifestações clínicas da doença são inespecíficas, e essa hipótese geralmente só é aventada após o acometimento de um órgão em particular. A idade média para a realização do diagnóstico é de 64 anos e ocorre ligeiro predomínio de ocorrência no gênero masculino $^{(1)}$. O termo amilóide foi criado por Virchow na metade do século XIX em estudos de autópsia, referindo-se às propriedades de coloração de depósitos no tecido hepático de substância semelhante ao amido, logo após a aplicação de iodo e ácido sulfúrico ${ }^{(2)}$. A nomenclatura de classificação da amiloidose é feita da seguinte forma: a primeira letra é sempre a "A", que corresponde à palavra amiloidose; a letra subseqüente é uma abreviação da natureza bioquímica

\section{ABSTRACT}

Amyloidosis is a rare disease caused by pathological deposit of an amyloid extracellular proteinaceus material. We report a case of a 50-year-old man with history of lips and tongue swelling associated to periorbital ecchimosys for 6 months. At the physical examination an important macroglossia was observed. Complementary tests were accomplished, and the pathological examination of the tongue biopsy confirmed the diagnosis. The patient was treated with prednisone and melphalan with an unsatisfactory response. Along the follow up the patient developed heart failure, and died 7 months after diagnosis of pulmonary infection and sepsis.

Keywords: amyloidosis, macroglossia, periorbital ecchimosys.

da fibrila encontrada ${ }^{(3)}$. As abreviaturas que designam os tipos de amiloidose com acometimento sistêmico são AL (imunoglobulina de cadeia leve = primária $), \mathrm{AA}$ (reativa; secundária) e $\mathrm{A} \beta 2 \mathrm{M}$ ( $\beta 2$ microglobulina, associada à diálise $)$. A amiloidose sistêmica pode comprometer as articulações, $\mathrm{O}$ que causa a confusão da mesma com doenças reumatológicas auto-imunes. Cerca de $95 \%$ das amiloidoses são do tipo AL ou AA. A amiloidose do tipo AA tem maior incidência entre os pacientes portadores de tuberculose, hanseníase e artrite reumatóide. Os órgãos mais acometidos são fígado, baço, rins e glândulas adrenais. O quadro clínico é iniciado por uma proteinúria associada à hepatoesplenomegalia, com evolução para síndrome nefrótica e doença renal terminal. Já a amiloidose AL (primária) é uma doença progressiva de prognóstico ruim, com deposição de amilóide em língua (12 a 20\% dos casos) $)^{(4)}$, coração, trato gastrintestinal,

\footnotetext{
Serviços de Reumatologia e de Cirurgia de Cabeça e Pescoço do Hospital Ana Costa, Santos. Recebido em 05/01/05. Aprovado, após revisão, em 30/10/06.

1. Acadêmica do Curso de Medicina da Universidade Metropolitana de Santos

2. Acadêmica do Curso de Medicina do Centro Universitário Lusíada, Santos.

3. Doutor em Medicina pelo Curso de Pós-graduação em Otorrinolaringologia e Cirurgia de Cabeça e Pescoço da Universidade Federal de São Paulo - Escola Paulista de Medicina (Unifesp/EPM).

4. Especialista pela Sociedade Brasileira de Reumatologia

5. Mestre em Anatomia Patológica pela Unifesp/EPM.

6. Médica residente de Clínica Médica do Hospital Ana Costa.

Endereço para correspondência: Carla de Oliveira Alambert, Rua Francisca Faria, 111, ap. 11, CEP 11035-250, Santos, SP, Brasil, e-mail: c.alambert@uol.com.br.
} 
sistema osteomuscular, nervos e pele. O quadro clínico inicia-se com fadiga e perda de peso, mas o diagnóstico é raramente realizado até que os sinais/sintomas relacionados a um órgão em particular apareçam. Outros tipos menos comuns são: amiloidose tipo ATTR, que se manifesta como amiloidose sistêmica senil, com envolvimento cardíaco, ou como polineuropatia familiar amilóide, com comprometimento cardíaco ou renal; amiloidose $\mathrm{A} \beta 2 \mathrm{M}$, em que ocorre depósito periarticular e eventual comprometimento renal; amiloidose AIAPP, na qual ocorre depósito de substância amilóide nas ilhotas de Langerhans, podendo manifestar-se clinicamente como diabetes tipo 2 e insulinoma; amiloidose AApoA, que está relacionada com polineuropatia familiar e nefropatia; amiloidose AGel, que cursa com polineuropatia familiar com lattice corneal dystrophy, neuropatia craniana e nefropatia; amiloidose AFib, na qual encontramos a amiloidose familiar com nefropatia; amiloidose ALys, cujas manisfestações são as mesmas da AFib, mudando apenas a composição da fibrila; amiloidose $A \beta$, que se relaciona com a doença de Alzheimer, com a síndrome de Down e com a angiopatia amilóide cerebral (Dutch); amiloidose ACys, cuja associação é com a angiopatia amilóide cerebral (Icelandic); amiloidose ACal, relacionada com o carcinoma medular de tireóide; e, por fim, a amiloidose AANF, cuja síndrome clínica manifesta-se por meio da amilóide atrial $\operatorname{localizada}^{(3)}$. A seguir, descrevemos um caso de amiloidose primário cujo diagnóstico foi suspeitado a partir da identificação de macroglossia.

\section{RELATO DE CASO}

Paciente de 50 anos, sexo masculino, caucasiano, casado, chega a nosso serviço com uma história de edema progressivo de lábios e língua há 6 anos. Relata que há 6 meses começou a apresentar piora do quadro com dificuldade de deglutição e fonação, evoluindo também com edema de membros inferiores, edema de região periorbitária e equimoses palpebrais bilaterais. Relata que vem fazendo uso de furosemida sem sucesso há 2 meses. Refere diagnóstico prévio de alergia a corantes e conservantes. Não apresentava antecedentes familiares patológicos.

Ao exame físico, o paciente encontrava-se febril (temperatura axilar $38^{\circ} \mathrm{C}$ ), pulso de $110 \mathrm{bpm}$, pressão arterial de $100 \times 70 \mathrm{mmHg}$, macroglossia, edema e equimose periorbitária bilateral, presença de linfonodos cervicais palpáveis bilateralmente (pequenos, móveis), fígado palpável a $3 \mathrm{~cm}$ do rebordo costal direito e edema de membros inferiores $++/ 4+$.
Os principais dados clínicos observados durante a avaliação desse paciente foram a macroglossia, a púrpura periorbitária, o edema e a hepatomegalia.

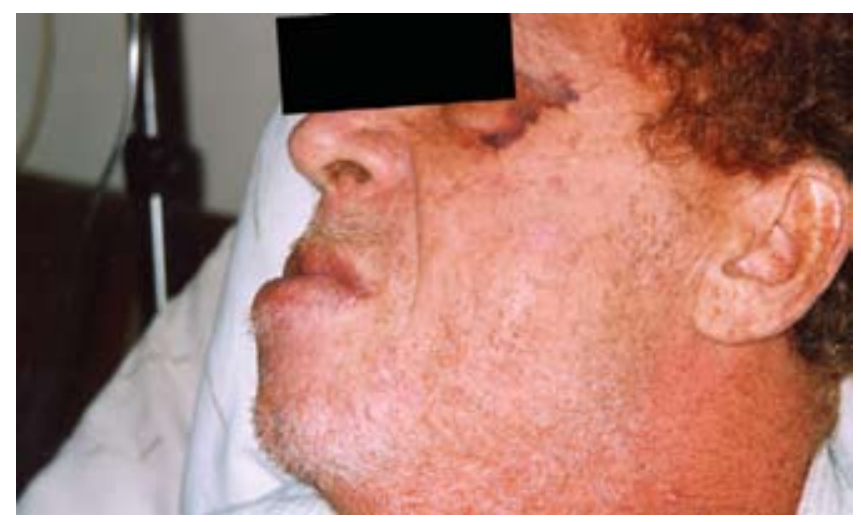

Figura 1 - Equimose periorbital.

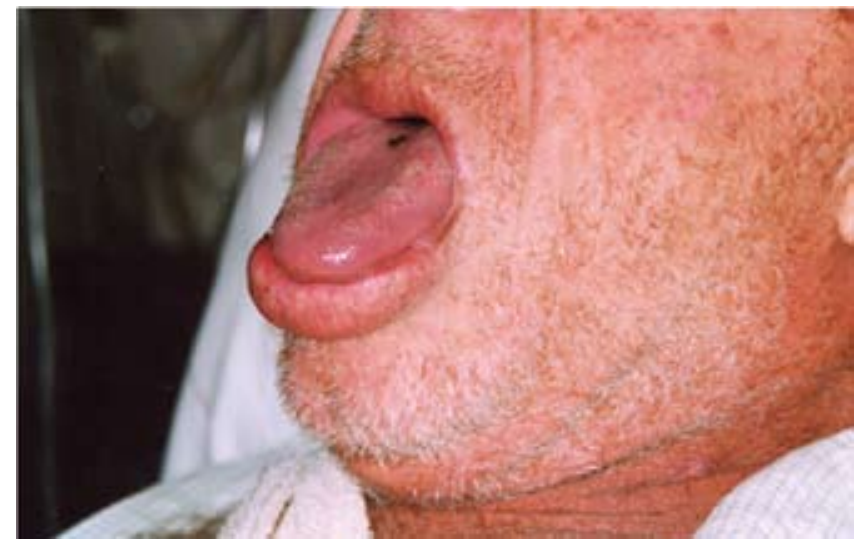

Figura 2 - Macroglossia.

Os exames complementares incluem: hemograma com

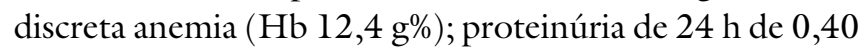
$\mathrm{g} / \mathrm{l}$ (valor de referência: até $0,08 \mathrm{~g} / \mathrm{l}$ ); eletroforese de proteínas séricas com hipoalbuminemia de $2,5 \mathrm{~g} / \mathrm{dl}$ (valor de referência: 3,0 a $5,2 \mathrm{~g} / \mathrm{dl}$ ). O eletrocardiograma mostrava fibrose anterosseptal (alteração freqüente em pacientes com amiloidose) e bloqueio divisional anterossuperior esquerdo. No ecocardiograma observava-se aumento de grau leve dos átrios, hipertrofia simétrica do ventrículo esquerdo de grau importante, desempenho contrátil conservado, espessamento das valvas mitral e aórtica; biópsia de língua evidenciando pesquisa positiva para amiloidose na luz polarizada e coloração vermelho-congo.

O paciente foi tratado com melfalan oral cíclico e prednisona (melfalan na dose de 0,15 a $0,25 \mathrm{mg} / \mathrm{kg} /$ dia e prednisona 1,5 a $2,0 \mathrm{mg} / \mathrm{kg} / \mathrm{dia}$ ) durante 4 dias. Apesar do tratamento, o paciente desenvolveu insuficiência cardíaca, 


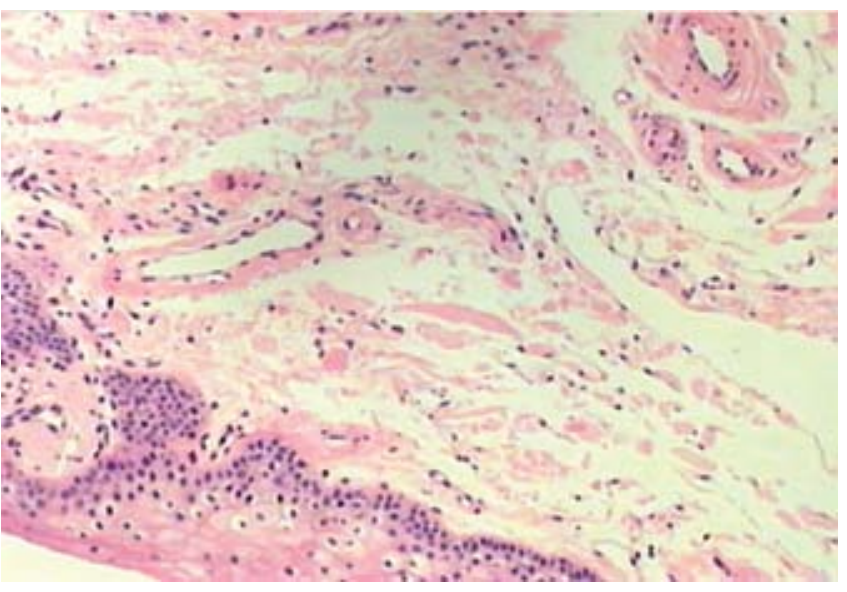

Figura 3 - Biópsia de língua com indicação de amiloidose pela luz polarizada.

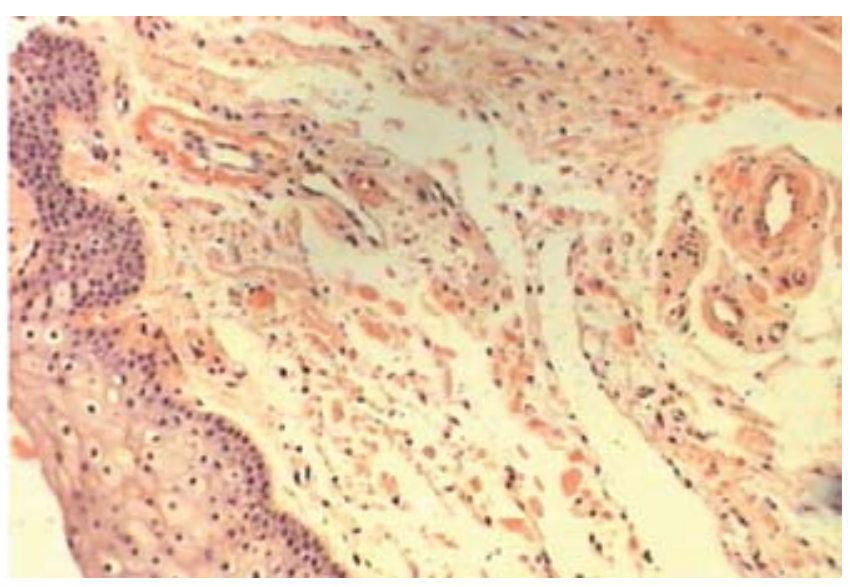

Figura 4 - Biópsia de língua e coloração por vermelho-congo.

proteinúria e infecções urinárias e pulmonares de repetição. Foi internado 7 meses após o diagnóstico com infecção pulmonar complicada com sepse, evoluindo a óbito.

\section{DISCUSSÃO}

Uma das primeiras manifestações da amiloidose primária pode ser somente o aumento do volume da língua ${ }^{(5)}$. $\mathrm{O}$ diagnóstico de amiloidose não deve ser o primeiro a ser aventado quando nos deparamos com um caso de macroglossia. Outras doenças, como tumor maligno de língua, doença vascular ou etiologia sistêmica, como hipotireoidismo ou deficiência de vitamina B12 ou de ácido fólico, devem ser sempre investigadas ${ }^{(6)}$.

A macroglossia encontra-se presente em 12 a $20 \%$ dos pacientes com amiloidose sistêmica primária; considera-se a amiloidose a primeira causa de macroglossia em adultos ${ }^{(4)}$. A púrpura também é um sinal freqüente em amiloidose primária, ocorre em até $40 \%$ dos casos e é conseqüente à infiltração amilóide na parede vascular; pode ocorrer no pescoço, face e olhos, sendo a lesão mais característica a púrpura periorbital ${ }^{(4)}$. A presença de edema ocorre em $30 \%$ dos pacientes e há hepatomegalia em 25 a $50 \%{ }^{(4)}$. Durante a evolução da doença, pode ocorrer síndrome nefrótica em $29,9 \%$ dos casos, insuficiência cardíaca congestiva em $21,9 \%$, síndrome do túnel do carpo em $21,5 \%$, neuropatia periférica em $17 \%$ e hipotensão ortostática em $12 \%$ dos pacientes ${ }^{(1)}$.

O paciente em questão apresentou proteinúria em nível inferior ao nefrótico, encontrada em $80 \%$ dos casos ${ }^{(1)}$. Evoluiu com sinais clínicos de acometimento cardíaco. Os pacientes sintomáticos, do ponto de vista cardiovascular, geralmente apresentam mais de $25 \%$ de comprometimento miocárdico ${ }^{(7)}$. $\mathrm{O}$ aumento de espessura da parede ventricular e do septo correlaciona-se com maior incidência de falência cardíaca $^{(1)}$. Sabe-se que a insuficiência cardíaca é observada em cerca de $20 \%$ dos pacientes no momento do diagnóstico e desenvolve-se com a evolução da doença em cerca de $10 \%$ dos casos. Cerca de $40 \%$ dos pacientes evoluem a óbito por insuficiência cardíaca congestiva ou arritmias ${ }^{(4)}$. Em aproximadamente $50 \%$ dos pacientes a eletroforese de proteínas séricas evidencia uma banda localizada ou pico de tamanho moderado (em média 1,4 g/dl). Hipogamaglobulinemia ocorre em cerca de $20 \%$ dos casos $^{(1)}$. No entanto, a eletroforese de proteínas séricas do paciente apenas evidenciou a presença de hipoalbuminemia.

Uma vez estabelecida a suspeita clínica de amiloidose, devemos confirmar o diagnóstico com a biópsia do órgão afetado e a avaliação histopatológica. A técnica utilizada é a de coloração pelo vermelho-congo, que foi introduzida por Bennhold em 1922; a substância amilóide caracteriza-se por uma coloração vermelho-alaranjada quando avaliada à luz normal, mas o diagnóstico é confirmado pela birrefringência de coloração esverdeada observada ao microscópio com luz polarizada ${ }^{(8)}$.

A média de sobrevida dos pacientes com amiloidose primária é de 13 meses, variando de acordo com a síndrome associada, sendo de apenas 4 meses após o início da insuficiência cardíaca. Os pacientes que apresentam apenas neuropatia periférica possuem sobrevida média de 2 anos $^{(1)}$.

A resposta ao tratamento geralmente é insatisfatória. $\mathrm{O}$ tratamento da amiloidose não dispõe até o momento de uma intervenção eficaz e segura para a maioria dos tipos. Todo planejamento deverá encaminhar-se para dois objetivos: diminuir a proteína precursora do tipo amilóide em questão e terapia de suporte para o órgão acometido pelo depósito da fibrila amilóide. 
O esquema de tratamento empregado (vide relato de caso) deve ser repetido cada 6 semanas por 1 ano para um total de $600 \mathrm{mg}$ de melfalan ${ }^{(9)}$. Por meio de um ensaio randomizado com 100 pacientes (nível III de evidência), concluiu-se que o tratamento com múltiplos agentes alquilantes e outros (vincristina, carmustina, melfalan, ciclofosfamida e prednisona) não melhorou a resposta $\mathrm{e}$ o tempo de sobrevida comparado com o tratamento com melfalan e prednisona ${ }^{(9)}$. Concluiu-se também, por um outro estudo, que a associação de melfalan e prednisona apresenta uma resposta terapêutica superior ao uso da colchicina ${ }^{(10)}$. Em um estudo caso-controle (nível V de evidên-

\section{REFERÊNCIAS}

1. Kyle RA, Gertz MA: Primary systemic amyloidosis: clinical and laboratory features in 474 cases. Semin Hematol 32: 45-59, 1995.

2. Diniz REA, Sementilli A, Dedivitis RA, et al: Amiloidose. Revista Médica Ana Costa 8: 7-24, 1998.

3. Seldin, DC, Skinner M: Amyloidosis. In: Harris ED, Budd RC, Firestein GS, Genovese MC, Sergent JS, Ruddy S, Sledge CB: Kelley's Textbook of Rheumatology. 7. ed. Philadelphia, 2005. p. 1697-1704.

4. Moroni L, Ana María Benavides M, Alicia y Retamal E, Yasmina: Macroglosia y amiloidosis oculta/Macroglossia as the presenting symptom of amyloidosis. Rev Méd Chile 130: 215-8, 2002.

5. Babajews A: Occult multiple myeloma associated with amyloid of the tongue. Br J Oral Maxillofac Surg 23: 298-303, 1985.

6. Xavier SD, Bussoloti Filho I, Müller H: Macroglossia decorrente de amiloidose sistêmica: relato de caso e revisäo de literatura/ cia) foi observado que ocorre maior sobrevida em pacientes com amiloidose sistêmica primária submetidos a PBSCT (peripheral blood stem cell transplantation) $)^{(11)}$.

Como parte do tratamento, devemos também promover o suporte clínico às possíveis complicações da doença. A síndrome nefrótica deve ser tratada com restrição de sal e diuréticos. Deve-se evitar o uso de digitálicos no tratamento da insuficiência cardíaca, pois esses pacientes são muito sensíveis a esses agentes, ocorrendo freqüentemente bloqueio átrio-ventricular e arritmias.

Declaramos a inexistência de conflitos de interesse.

Macroglosia due to sistemic amyloidosis: case report and literature review. Rev Bras Otorrinolaringol 70: 715-9, 2004.

7. Spirito P, Seidman CE, McKenna WJ, Maron BJ: The manegement of hypertrophic cardiomyopathy. N Eng J Med 336: 775-85, 1997.

8. Stoopler ET, Sollecito TP, Chen SY: Amyloid deposition in the oral cavity: a retrospective study and review of the literature. Oral Surg Oral Med Oral Pathol Oral Radiol Endod 95: 674-80, 2003. verificar nome da revista???

9. Skinner M: Amyloidosis. In: Lichtenstein LM, Fauci AS. Current Therapy in Allergy, Immunology and Rheumatology. Philadelphia: Mosby-Year Book, 1996. p. 235-240.

10. Kyle RA, Gertz MA, Greip PR, et al: A trial of three regimens for primary amyloidosis: colchicine alone, melphalan and prednisone, and melphalan, prednisone, and colchicine. N Eng J Med 336: 1202-7, 1997.

11. Dispenzieri A: High-dose therapy for amyloidosis: the end of the beginning? Blood 103: 3612-13, 2004. 